\title{
FOUCAULT: ROSTRO DE ARENA, DISCONTINUIDAD HISTÓRICA Y “JEUX DE VÉRITÉ”
}

\author{
FOUCAULT: A FACE DRAWN IN SAND, HISTORICAL \\ DISCONTINUITY, AND "JEUX DE VÉRITÉ"
}

\author{
Antonia Tejeda Barros*
}

\begin{abstract}
Resumen
La muerte del hombre anunciada por Foucault está en estrecha relación con la muerte de Dios anunciada por Nietzsche. El retorno del lenguaje conlleva la desaparición del hombre como figura cardinal del saber moderno. La historia es discontinua. Foucault dudó de las verdades de cada época y de las verdades intemporales. En este artículo trazo el recorrido del hombre en Les mots et les choses, mostrando la tensión entre el lenguaje y la desaparición del hombre, hablo de la discontinuidad de la historia y los "jeux de vérité", y muestro que Foucault se consideraba, por encima de todo, un pensador escéptico.
\end{abstract}

Palabras clave: arqueología, genealogía, ciencias humanas, teoría crítica, discontinuidad, escepticismo, "jeux de vérité".

\begin{abstract}
The death of man proclaimed by Foucault is in close relationship with the death of God proclaimed by Nietzsche. The return of language is linked with the disappearance of man. History is discontinuos. Foucault doubted the truths of each era as well as the timeless truths. In this article I present man's journey in Foucault's Les mots et les choses, showing the tension between language and the disappearance of man, I talk about historical discontinuity and the "jeux de vérité", and I argue that Foucault considered himself, above all, a skeptical thinker.
\end{abstract}

Keywords: archeology, genealogy, human sciences, critical theory, discontinuity, skepticism, "jeux de vérité".

Recibido: 2020-05-28 Aceptado: 202I-02-24

* Magíster en Filosofía. Doctoranda en Filosofía, UNED. Director de tesis: Manuel Fraijó Nieto. E-Mail: antoniatejedabarros@gmail.com. 


\section{Introducción}

"Foucault est fondamentalement, dans l'ordre de la philosophie de la connaissance, un penseur sceptique qui ne croit pas à l'existence d'une vérité objective" (Quiniou 20I4).

La obra de M. Foucault se centra en el lugar que va ocupando el hombre en la historia a través de los saberes, de los discursos, de las técnicas de conocimiento y de las prácticas de poder. Foucault descubrió a F. Nietzsche hacia I953 y este descubrimiento jugó un papel importantísimo en su ruptura con la fenomenología y el existencialismo (ambos centrados en el sujeto). La genealogía foucauldiana es una explicación causal histórica que es material, múltiple y corpórea. La genealogía de Nietzsche opera con causas psicológicas (el resentimiento de los débiles, la ambición de los fuertes) que poco tienen que ver con la historia del cuerpo de la que habla Foucault. Así pues, como metodologías históricas, las genealogías de Nietzsche y Foucault son muy distintas, aunque se asemejan por su poder crítico y su relación entre el conocimiento y el poder (Gutting 2005a).

Aunque Foucault no se consideró estructuralista, existen afinidades entre el estructuralismo y la arqueología de Foucault. J. Piaget (I968) define el estructuralismo de Foucault como un estructuralismo sin estructuras. La superación estructuralista del sujeto es en Foucault una temática epistemológica y política. F. Rambeau afirma que, a pesar de todas las etiquetas que Foucault recibió (estructuralista, antihumanista, neomarxista, postmoderno), Foucault fue ante todo un pensador inclasificable (Rambeau 2006). P. Veyne asevera que Foucault no fue un pensador estructuralista ni relativista ni historicista ni nihilista; fue, ante todo, un pensador escéptico. ${ }^{\mathrm{I}}$

\section{El nacimiento y la muerte del hombre}

"L’homme est une invention dont l'arquéologie de notre pensée montre aisément la date récente. Et peut-être la fin prochaine" (Foucault 2013, p. 398).

La historia que atrae a Foucault es la historia de las problematizaciones. La episteme de finales del siglo XVIII-principios del siglo XIX ve aparecer por primera vez la figura del hombre en el campo del saber: el

I "Non, Foucault ne fut pas un penseur structuraliste (...) Chose rare en ce siècle, il fut, de son prope aveu, un penseur sceptique (...) Ce ne fut pas un nihiliste pour autant: il constatait l'existence de la liberté humaine" (Veyne 2010, p. 9). 
hombre aparece en el seno del pensamiento y del saber moderno y es, a la vez, objeto (empírico) y sujeto (trascendental) del conocimiento. La ontología histórica de nosotros mismos muestra cómo nos constituimos en sujetos de conocimiento (sujetos epistemológicos), sujetos de acción (sujetos de poder) y sujetos morales en cada una de las épocas ${ }^{2}$. Foucault indica, pues, que existen tres dominios de genealogía posibles ${ }^{3}$. El sujeto no es, para Foucault, una substancia, sino una forma, y esta forma (que no es ni universal ni abstracta) varía dependiendo del contexto sociohistórico (Lahire 20I3). Contra las filosofías del sujeto, Foucault pretende mostrar cómo el hombre se constituye en la historia a través de los saberes, de los discursos, de las técnicas de conocimiento y de las prácticas de poder. ${ }^{4}$

Los objetos de conocimiento están constituidos por un conjunto de ideas, esquemas y prácticas que Foucault llama discurso. El discurso nos da una perspectiva histórica condicionada que no nos descubre la esencia de los hechos históricos sino que los interpreta (Quiniou 2009). Nos encontramos, pues, con un Foucault escéptico que no apuesta por una verdad universal sino por juegos de verdad: "il n'y a donc pas de vérité objective susceptible de se développer dans le temps par accumulation; il n'y a que des «jeux de vérité»» (Quiniou 2009). Así pues, la enfermedad mental no es una realidad objetiva sino una construcción de la psiquiatría moderna. La sexualidad deviene, históricamente, objeto de diferentes tópicos: es objeto del placer en la Antigüedad, objeto de la carne en la Edad Media y objeto de la libido en el psicoanálisis (Quiniou 2009) (ninguna de estas definiciones de sexualidad -objeto del placer, objeto de la carne y objeto de la libido-nos revela la verdad de la sexualidad, sino que solamente describe la sexualidad en cada época histórica concreta). La verdad no es libre y está vinculada a las relaciones de poder. En Histoire de la sexualité, vol. I. La volonté de savoir, Foucault describe cómo el sexo dejó de ser, en un momento histórico, una cuestión de placer y

2 «Ésos son los tres campos que estudiará a lo largo de toda su obra Foucault: a) sujetos de conocimiento ... b) sujetos de acción ... c) sujetos morales" (Savater 2009, p. 296).

3 "une ontologie historique de nous-mêmes dans nos rapports à la verité, qui nous permet de nous constituer comme sujets de connaisance; dans nos rapports à un champ de pouvoir, qui nous permet de nous constituer comme sujets agissants sur les autres; et dans nos rapports à la morale, qui nous permet de nous constituer en agents éthiques" (Revel 2009, p. 62).

4 'Cet 'antihumanisme' fit scandale et le rapprochait du structuralisme, lequel critiquait lui aussi de manera radicale les philosophies qui, de Descartes à ... Sartre, appréhendaient le sujet comme une conscience libre, autoconstituée et antihistorique" (Halpern 2013, p. 73). 
sensación, y pasó a convertirse en una cuestión de verdad y falsedad (sexo heterosexual versus sexo homosexual).

Los modos de pensamiento de una época están destinados a perecer para ser reemplazados por otros. "Avant du XIX siècle en peut dire que l'homme n'existait pas”, afirma Foucault (Dumayet I966). En lugar del hombre existían formas de saber y de reflexión: reflexión sobre Dios, sobre la Naturaleza, sobre la virtud, sobre el orden. El hombre aparece a finales del siglo XVIII-principios del siglo XIX y da lugar a las ciencias humanas y al humanismo. Y de la misma manera que el hombre nace como objeto de conocimiento (cuando nacen las ciencias humanas), así el hombre desaparecerá en un futuro próximo (con el retorno del lenguaje) como objeto de conocimiento. ${ }^{5}$

El psicoanálisis y la etnología ocupan un lugar privilegiado en el área del saber: ambos acumulan experiencias y conceptos sobre el hombre y ambos se interrogan sobre el saber del hombre. El psicoanálisis habla desde el inconsciente y desarrolla una función crítica. La etnología habla desde la historicidad. Foucault asevera que la lingüística, el psicoanálisis y la etnología ya no hablan del hombre, sino que lo disuelven. En lugar de ciencias son contra-ciencias. ${ }^{6}$ Este es el principio del fin del hombre.

El hombre queda definido por aquello que lo limita: por la muerte (como destino de la vida), por la alienación (como destino del trabajo) y por la opacidad del lenguaje (como destino del lenguaje). El cuerpo, el deseo y el lenguaje son las formas fundamentales de su finitud. ${ }^{7}$ El nacimiento del hombre implica también su inevitable muerte. La muerte del hombre es una muerte epistemológica: el hombre morirá como sujeto, como agente de la historia. El hombre aparece cuando el discurso se apaga y el hombre morirá cuando el ser del lenguaje reaparezca.

Foucault sostiene que no hay verdades permanentes: todo cambia a lo largo de la historia. Las ideas tienen su genealogía, su desarrollo y su muerte. La idea de hombre tiene, pues, también, su propia genealogía y

5 "Quand Foucault parle de la mort de l'homme, il faut immédiatement préciser que c'est toujours en relation avec sa très récente apparition ('avant la fin du XVIIIe siècle, l'homme n'existait pas'). Car c'est de l'homme comme figure, pli du savoir qu'il s'agit" (Gros 20I2, p. 4I).

6 "Par rapport aux "sciences humaines", la psychanalyse et l'ethnologie son plutôt des "contre-sciences"... elles ne cessent de "défaire" cet homme qui dans les sciences humaines fait et refait sa positivité" (Foucault 2013, p. 39I).

7 "À travers [des] figures de la Mort, du Désir, de la Loi, qui schématisent aux yeux de Foucault le dispositif freudien (articulé autour de la pulsion de mort, de l'économie libidinale et de la rhétorique de l'inconscient), le discours de l'insconscient se présente ... comme le discours de l'impensé" (Sabot 2006, p. I66). 
su propio fin. Nietzsche anunció la muerte de Dios. Foucault anuncia la muerte del hombre. Y esta muerte es anunciada de una manera poética en las últimas famosas palabras de Les mots et les choses: "L'homme s'effacerait, comme à la limite de la mer un visage de sable" (Foucault 2013, p. 398).

\section{La discontinuidad histórica}

La historia del pensamiento, del conocimiento, de la filosofía y de la literatura es discontinua. Foucault analiza la historia dentro de una discontinuidad y la despoja de todo "narcisismo trascendental" (Foucault llamó burlonamente "narcisismo trascendental" a la filosofía centrada en el sujeto de pensadores como Sartre). Foucault no habla de una historia del espíritu, sino de una historia de los discursos, los cuales constituyen la descripción más precisa de la formación histórica. ${ }^{8}$ La episteme de cada época está constituida por las relaciones entre los diferentes discursos científicos. ${ }^{9}$ Foucault recompone las piezas culturales de cada época, sacando a flote los discursos que tienen como paradigma un saber concreto.

Foucault afirma que el pensamiento clásico tiene una relación estrecha con el infinito: la forma que predomina no es la forma-hombre, sino la forma-Dios (la forma de lo infinito). Foucault introduce los conceptos de pli (pliegue) y dépli (despliegue) para explicar esta relación de fuerzas hacia lo infinito (dépli) y hacia lo finito (pli). En el siglo XIX desaparece el discurso y las fuerzas del hombre entran en relación con nuevas fuerzas de finitud (la vida, el trabajo y el lenguaje) -estas fuerzas harán nacer la biología, la economía política y la lingüística. Con las nuevas formas de finitud (vida, trabajo, jenguaje) aparece la forma-hombre en substitución de la forma-Dios. La finitud del hombre reemplaza la infinitud de Dios. El nacimiento del hombre como sujeto acontece cuando se apaga la transparencia del discurso y las ciencias humanas surgen cuando aparece el hombre como saber positivo. Foucault sostiene que el

8 "Le fait que je considère le discours comme une série d'événements nous place automatiquement dans la dimension de l'histoire (...) Je ne suis pas un historien au sens strict du terme, mais les histoires et moi avons en commun un intérêt pour l'événement" (Revel 2009, p. 5I).

9 Foucault declara en Les mots et les choses que existen tres grandes períodos en la historia del pensamiento occidental. A cada período le corresponde una episteme (objeto de conocimiento). En el Renacimiento el saber se funda bajo el concepto de semejanza (ressemblance). En la edad clásica (siglos XVII y XVIII) aparece una nueva relación entre las palabras y las cosas, se separa el significante del significado y el saber toma como referencia el concepto de representación. En la época moderna (finales del siglo XVIII-principios del siglo XIX) aparece una nueva episteme: el hombre. 
humanismo no nace en el Renacimiento, sino a finales del siglo XIX: "Le mot humanisme est une invention du XIXe siècle" (Dumayet I966).

A pesar de que la obra de Foucault escape cualquier etiqueta, la mayoría de sus libros pueden clasificarse como historias (Gutting 2005a). En L'archéologie du savoir, Foucault confiesa: "Au fond je ne suis peut-être qu'un historien des idées" (I84). La historia de las ideas se centra en los márgenes y analiza más bien los errores que la verdad. Foucault dijo una vez: "I am not a professional historian; nobody is perfect". ${ }^{\circ}$ Entre el análisis arqueológico y la historia de las ideas hay muchas afinidades, pero también algunas diferencias importantes. La arqueología intenta definir los discursos; es una reescritura (Foucault 20I2b).

Los métodos arqueológico y geneaológico foucauldianos se definen por la inversión de perspectiva, la marginalidad y la discontinuidad. La historia no tiene ni sentido ni finalidad. El progreso no existe. Los hechos

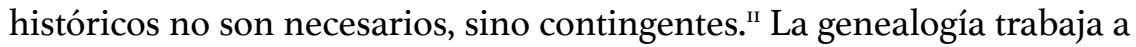
partir de la diversidad, la dispersión, la coincidencia y los hechos accidentales, y no busca reestablecer la continuidad de la historia, sino restituir los sucesos en su singularidad (Revel 2009). La historia, pues, no es una progresión de eventos interconectados sino un conjunto de discontinuidades. Jean-Luc Godard ridiculizó esta idea foucauldiana de discontinuidad en La Chinoise: Jean-Pierre Léaud interpreta a un estudiante fanático maoísta que en un momento le tira flechas a varios libros, y entre ellos se encuentra Les mots et les choses. ${ }^{12}$

\section{Escéptico, pero no enemigo de la humanidad}

«[Foucault] fut ... un penseur sceptique, qui ne croyait qu’à la vérité des faits, des innombrables faits historiques qui remplissent toutes les pages de ses livres» (Veyne 20I0, p. 9).

En Foucault. Sa pensée, sa personne, Veyne define a Foucault como un pensador escéptico: «sceptique, mais pas ennemi de l'humanité» (Veyne

Io Charla en la University of Vermont (EEUU) el 27 de octubre de 1982 (Gutting 2005b).

II "Foucault aimed at revealing the historical contingency of ideas that present themselves as necessary, unsurpassable truths" (Gutting 2005b).

I2 Godard llamó a Foucault Le Révérend Père Foucault y dijo en una entrevista: «Si je n'aime pas ... Foucault, c'est parce qu'il nous dit: 'À telle époque, les gens pensaient ceci ou cela, et puis à partir de telle date, on a pensé [ceci ou cela]' (...) C'est justement pour ça que nous tentons de faire des films: pour que des Foucaults futurs ne puissent affirmer de telles choses avec autant de présomption» (Eribon 20II, p. 267). 
20IO, p. 7I). El escepticismo de Foucault no es una postura que duda de la realidad de los hechos históricos, sino que es un escepticismo que se deja notar en las grandes preguntas: ¿qué es la verdadera democracia? ¿qué es el verdadero poder? Foucault no produjo una teoría global del poder ni un sistema ético ni un sistema histórico. El trabajo filosófico de Foucault fue un trabajo de desidentificación. ${ }^{13}$

Las ciencias humanas no descubren el núcleo concreto, individual y positivo de la existencia humana. Al estudiar la historia de nuestro saber, nos damos cuenta de que el núcleo no es el hombre en su positividad, sino los sistemas de pensamiento. Foucault sostiene que, para analizar la historia del conocimiento, no es preciso pasar por el hombre. Ni el hombre como objeto empírico ni el hombre como sujeto trascendental son necesarios para la historia del saber. Cada una de las ciencias humanas estudia un aspecto del hombre sin pretender contribuir a una concepción general del hombre (Veyne 20I0).

La ausencia del hombre en el saber clásico queda reflejada en el análisis que Foucault hace de Las Meninas en el primer capítulo de Les mots et les choses. Las Meninas representa para Foucault un punto de entrada y de salida en el Renacimiento. Toda la composición del cuadro (la posición del pintor, las miradas de los personajes, la luz) gira en torno a un personaje (el rey) que se encuentra ausente en el cuadro (apenas se ve su reflejo en el espejo, junto a la reina): el hombre, como sujeto de la representación, está ausente (Sabot 2006). Esta ausencia del sujeto en Las Meninas representa para Foucault la equivalencia pictórica de la ausencia del hombre en la teoría clásica del saber.

Foucault sostiene que el sujeto humano no puede hacer bajar del cielo una verdad absoluta ni tampoco actuar en el cielo de las verdades (Veyne, 20Io); lo único que puede hacer es actuar contra las verdades y las realidades de su propia época. Este sujeto no es ni soberano ni absoluto: "le sujet n'est pas 'naturel', il est modélé à chaque époque par le dispositif et les discours du moment" (Veyne 20IO, p. I72). No existe un sujeto universal ni tampoco una moral universal. La moral universal es imposible, asevera Foucault. ${ }^{14}$

I3 «À cette «déprise de soi〉 Foucault donna, courageusement, la forme d`une fidelité à soi-même: la mise à l’épreuve sans cesse reconduite de notre capacité vitale à nous modifier» (Rambeau 2006, p. 86).

I4 «Il en arrive même, dans Les Mots et les Choses à nouveau, à déclarer que toute morale universelle est « impossible » et tout ce qu'il en affirme dans ses Dits et écrits va rigoureusement dans le même sens» (Quiniou 2014). 
Foucault no busca una verdad universal, sino que intenta descubrir contingencias que siempre han estado disfrazadas de necesidades. Su proyecto no es trascendental sino genealógico (en su diseño) y arqueológico (en su método) (Gutting 2005a). Veyne define el pensamiento de Foucault como un escepticismo original y como una antropología empírica fundada en la crítica histórica. Foucault dudó de la metafísica, la antropología filosófica y la filosofía moral: "Paix aux petits faits, guerre aux généralités", así resume Veyne (2010) la máxima foucauldiana: positivista en el detalle pero escéptica en su totalidad (Pol-Droit 2008).

La verdad no existe; lo único que existe es lo que creemos en un momento dado. Así define Veyne el escepticismo de Foucault. Foucault fue un escéptico, pero ello no significa que Foucault dudara de todo. Foucault creía en las pequeñas verdades, en los hechos: conocía el pensamiento griego y el pensamiento romano, no dudó de la inocencia de Dreyfus ni de la existencia de las cámaras de gas, pero admitió que era imposible saber qué era el porvenir y qué era la humanidad (Veyne 20I0). Existen singularidades, no totalidades.

P. Boucheron sostiene que Veyne ha hecho de Foucault "le portrait d'un sceptique" (2016). Boucheron admite que la obra de Foucault es ambigua y esto hace que cada uno pueda amoldar el pensamiento de Foucault a su propia idea. Boucheron afirma que aquellos que creen que Foucault se dedicó a destruir la verdad filosófica están equivocados, pero apunta que, en su último curso en el Collège de France, Foucault sostuvo que el filósofo busca la verdad de una manera heroica y escéptica.

Y. Quiniou (2009) afirma que la obra de Foucault ha suscitado muchos malentendidos y que Veyne ha sido uno de los pensadores que más ha contribuido a disipar estos malentendidos. Quiniou afirma que Foucault rechazó el concepto de una verdad objetiva y Veyne sostiene que Foucault fue un escéptico convencido (influido por el pragmatismo norteamericano, Wittgenstein y Nietzsche).

\section{La muerte de Dios, el Übermensch y Foucault}

"Nietzsche, me disait [Foucault], n'était pas un philosophe de la vérité, mais du dire-vrai" (Veyne 20I0, p. 157).

S. Royo, en una entrevista junto a T. Oñate (20I0), describe la muerte del hombre en la postmodernidad tras la muerte de Dios:

Ya Foucault en [Les mots et les choses] termina subrayando esta vinculación entre el humanismo moderno y la tradición teológica que primero puso a Dios en los altares y luego al hombre en el lugar de Dios (...) El sujeto mo- 
derno se desmorona con la crítica posthumanista ... que vino a promover la consideración de un sujeto múltiple [con] todos sus aspectos y toda su complejidad (p. 93).

La muerte del hombre anunciada por Foucault está en estrecha relación con la muerte de Dios anunciada por Nietzsche 84 años antes. Nietzsche mata a Dios con su famosa frase "Gott ist todt". ${ }^{15}$ Nietzsche sostiene que el hombre es una cuerda entre el animal y el Übermensch. El hombre es un ser que debe transformarse, superarse, un puente y no una meta. ${ }^{16}$ El superhombre nietzscheano es un hombre en el que todos sus valores se han transfigurado, un hombre con un espíritu fuerte y libre, con voluntad de poder, con una moral aristocrática; un hombre que dice sí a la vida, sí al instinto, sí al arte; un hombre no domado, un niño. Ortega dirá en La rebelión de las masas que la vida es un naufragio y el arte es la salvación. Nietzsche afirma que en el arte es donde se manifiestan los instintos, y que el artista es un hombre superior. ${ }^{17} \mathrm{El}$ superhombre abraza la totalidad: la vida y la muerte, la salud y la enfermedad, lo bueno y lo malo. El superhombre desprecia a Dios y dice sí a la vida, sí al mundo, sí al coraje, sí a la libertad. La muerte de Dios y la transvaloración de los valores darán paso al superhombre. Nietzsche mata a Dios y vislumbra la muerte del hombre: el hombre desaparecerá cuando llegue el superhombre: "Der Mensch ist ein Ende", exclama Nietzsche en Der Antichrist (\$3).

L. Feuerbach, K. Marx, Nietzsche y S. Freud anunciaron la muerte de Dios para dar paso al hombre. Feuerbach nació en I804 (I4 años antes que Marx, 40 años antes que Nietzsche y 52 años antes que Freud) y murió en I872, 29 años después de que Marx escribiera su famosa frase: "Die Religion ... ist das Opium des Volks"18 y II años antes de que Nietzsche publicara el primer libro de Also sprach Zarathustra. Freud consideró la

I5 Die fröhliche Wissenschaft ( $\$$ I08, 125 y 343) y Also Sprach Zarathustra ("Zarathustra's Vorrede", "Von den Mitleidigen" y "Ausser Dienst").

I6 "Was gross ist am Menschen, das ist, dass er eine Brücke und kein Zweck ist: was geliebt werden kann am Menschen, das ist, dass er ein Übergang und ein Untergang ist" (Nietzsche, KSA 4: I, $\$ 4$, pp, I6-17).

I7 Nietzsche critica a los filósofos por haber creado un lenguaje antiartístico: el lenguaje debe ser poetizado. La música compensa el efecto alienante que da el lenguaje común.

I8 La cita es parte de Zur Kritik der Hegelschen Rechtsphilosophie y se publicó en I844 en el Deutsch-Französische Jahrbücher: "Das religiöse Elend ist in einem der Ausdruck des wirklichen Elendes und in einem die Protestation gegen das wirkliche Elend. Die Religion ist der Seufzer der bedrängten Kreatur, das Gemüth einer herzlosen Welt, wie sie der Geist geistloser Zustände ist. Sie ist das Opium des Volks" (Marx 2016, pp. 12-13). 
religión como sentimiento de culpabilidad (Totem und Tabu), como delirio colectivo (Massenpsychologie und Ich-Analyse), como una idea delirante (Die Zukunft einer Illusion), como neurosis colectiva (Das Unbehagen in der Kultur) y como regresión e infantilismo (Der Mann Moses und die monotheistische Religion). Foucault irá más allá y anunciará, tras la muerte de Dios, la muerte del hombre. ${ }^{19}$

Cuando los filósofos mataron a Dios, tanto la moral y la política se tambalearon, pero ambas surgieron con más fuerza que nunca, asegura Foucault. Antes del siglo XIX, la filosofía se preguntaba qué moral y qué política se podían deducir a partir de la existencia de Dios. En el momento en que la filosofía y la cultura mataron a Dios, la moral y la política emergieron como entidades autónomas: "Jamais la réflexion morale, jamais la réflexion politique n'avaient été plus vivantes" (Dumayet, 1966).

Foucault afirma que la muerte de Dios y la muerte del hombre van unidas. El último hombre ha matado a Dios y debe responder de su propia finitud. El hombre, como asesino de Dios, está condenado a desaparecer: "L'homme va disparaître. Plus que la mort de Dieu ... ce qu'annonce Nietzsche, c'est la fin de son meurtrier" (Foucault 2013, p. 396).

Foucault sostiene que las grandes verdades se nos escapan. El escepticismo de Foucault es, sin embargo, un escepticismo alegre: el duelo de la verdad no le impide a Foucault ni reír ni actuar: "Un enfant de Nietzsche, évidemment" (Pol-Droit 2008).

\section{El fin de la filosofía}

"Il me semble que maintenant la philosophie est en train de disparaître", (Dumayet 1966).

Foucault afirma en i966 que la filosofía está desapareciendo. Hasta Sartre (inclusive) la filosofía fue una disciplina abierta que tenía un método propio, pero después de Sartre la filosofía empieza a disolverse. La filosofía se disipa en toda una serie de actividades del pensamiento que son difíciles de clasificar como propiamente científicas o propiamente filosóficas: la lingüística, la lógica, la literatura después de Joyce. Estas actividades del pensamiento no toman el lugar de la filosofía, pero sí aparecen como la base sobre la cual se desarrolla lo que antes fue la filosofia (Dumayet 1966).

19 "De nous jours, et Nietzsche là encore indique de loin le point d'inflexion, ce n'est pas tellement l'absence ou la mort de Dieu qui est affirmée mais la fin de l'homme" (Foucault 2013, p. 396). 
Foucault afirma en Les mots et les choses que la filosofía está a punto de desaparecer. ${ }^{20} Y$ con el fin de la filosofía asistimos irremediablemente a la desaparición del hombre. La desaparición del hombre está fuertemente vinculada a la recomposición del lenguaje. El hombre empieza a perecer a medida que brilla más el ser del lenguaje ("lêtre du langage") (Foucault 20I3). La literatura representa para Foucault el ser del lenguaje. Este ser del lenguaje no puede definirse por conceptos, puesto que no se trata de un objeto teórico, sino de una experiencia: la experiencia de la escritura. Foucault recurre a la imagen del espejo para designar la literatura: cuando las palabras no hablan más que de palabras y el lenguaje no habla más que de lenguaje nos encontramos con lo infinito de la literatura (Gros 2012).

Si la figura del hombre fue impuesta en el umbral de la modernidad en lugar del discurso clásico, el pensamiento contemporáneo (con su preocupación por el ser del lenguaje, sus sistemas simbólicos y sus estructuras) conllevará inevitablemente la disolución del hombre. Para Nietzsche la nueva fuerza que surge tras la muerte de Dios y la muerte del hombre es el Übermensch. Para Foucault la nueva fuerza que surge tras la muerte del hombre es el ser del lenguaje. La literatura muestra la reaparición del ser vivo del lenguaje ("lêtre vif du langage") (Foucault 2013).

Sartre se preocupó por aquello que es absolutamente auténtico en la existencia humana; ${ }^{21}$ Foucault pretende hacer precisamente lo contrario y afirma que la singularidad del hombre se encuentra más allá de un gran sistema formal. El pensamiento actual debe reconstruir este sistema formal sobre el cual flota de vez en cuando la imagen de nuestra propia existencia (Dumayet 1966).

\section{Conclusión}

El hombre es, según Foucault, una invención reciente en el campo del saber; nace a finales del siglo XVIII-principios del siglo XIX, junto a las ciencias humanas, y su fin puede vislumbrarse. La aparición del hombre es fruto de la fragmentación del lenguaje moderno. Hasta el siglo XVI el estudio del mundo se basa en la semblanza y la inter-

20 "le fait que la philosophie soit toujours et encore en train de finir et le fait qu'en elle peut-être, mais plus encore en dehors d'elle et contre elle, dans la littérature comme dans la réflexion formelle, la question du langage se pose, prouvent sans doute que l'homme est en train de disparaître" (Foucault 2013, p. 397).

2I "[Sartre] a voulu ramener l'homme à lui-même" (Duyamet I966). 
pretación. En el siglo XVII el estudio del mundo se basa en la representación y el orden. A finales del siglo XVIII-principios del siglo XIX la figura del hombre reemplaza el discurso clásico en el campo del saber. Foucault concibe al hombre como un pliegue en el saber (Gros 2012). «Jusqu'à la Revolution Française jamais on ne s'occuper de l'homme com tel» (Dumayet 1966). La muerte del hombre, pues, se encuentra en estrecha relación con la reciente aparición del hombre. ${ }^{22}$ Las desoladoras palabras con la que Foucault concluye Les mots et les choses señalan que no podemos interrogar el ser del hombre (como hizo Heidegger) ni su interioridad (como hizo Sartre) (Veyne 20Io).

Foucault describe al hombre casi como una pesadilla. El hombre es una invención y una figura determinada por la historia y situada en el interior de nuestra propia cultura. ${ }^{23} \mathrm{El}$ hombre fue constituido cuando el lenguaje estaba disperso y fragmentado. Cuando el lenguaje se recomponga, el hombre se dispersará. ${ }^{24}$ Foucault declaró, al igual que Montaigne y al contrario que Heidegger, que no tenemos ninguna comunicación con el ser. En L'archéologie du savoir, Foucault apunta: "Là où l'on fait parler les signes, il faut bien que l'homme se taise" (p. 275). La figura del hombre no es eterna; el hombre es un pliegue en nuestro saber que está condenado a desaparecer. El retorno del lenguaje conlleva la desaparición del hombre como figura cardinal del saber moderno.

Foucault rechaza la idea de que el saber tiene un desarrollo continuo (Halpern 20I3) y asevera que los modos de pensamiento de una época están destinados a perecer para ser reemplazados por otros. ${ }^{25} \mathrm{En}$ Les mots et les choses, Foucault sostiene que el hombre no ha sido a lo largo de la historia el problema central del saber humano. En medio de todas las mutaciones que han afectado el saber de las cosas y el orden de las cosas (Lordre des choses fue el título que Foucault había escogido originalmente para Les mots et les choses), ${ }^{26}$ en medio de todos los cam-

22 “Avant la fin du XVIIIe siècle, l'homme n'éxistait pas. Non plus que la puissance de la vie, la fécondité du travail, ou l'épaisseur historique du langage" (Foucault 2013, p. 3I9).

23 "Je crois que l'homme a été, si non un mauvais rêve, si non un cauchemar, du moins une figure très particulière, déterminé historiquement, située à l'intérieur de notre propre culture" (Dumayet 1966).

24 “L’homme s'étant constitué quand le langage était voué à la dispersion, no va-t-il pas être dispersé quand le langage se rassemble?" (Foucault 2013, p. 397).

25 "Foucault tire une conclusion majeure de ce mouvement de la pensée: les sciences humaines correspondent à un moment donné de l'histoire de notre savoir et il est fort possible que, dans l'avenir, l'homme s'effacera en tant qu'objet de connaissance, 'comme à la limite de la mer un visage de sable" (Lallement 2013, p. 35).

26 El título original fue usado finalmente en la traducción inglesa: The Order of 
bios que han afectado el saber de las identidades y diferencias aparece el hombre como pliegue del saber moderno apenas hace unos pocos siglos (Foucault 20I3). La figura del hombre se disuelve hoy en la literatura. Se anuncian, pues, nuevas formas de saber (la literatura y el ser del lenguaje). Foucault declara que lo único que podemos saber con certeza es que, en la cultura occidental, el ser del hombre y el ser del lenguaje jamás han podido coexistir ${ }^{27}$. La precariedad y muerte del hombre como saber está en estrecha relación con el retorno del ser del lenguaje..$^{28}$

Foucault escribió sobre historia, psicología, sociología, política, derecho y ética. Su obra se centra en el saber, el discurso, el poder, la sexualidad y la libertad. ${ }^{29}$ Foucault dio voz a los excluidos y marginados y fue el maestro de las ciencias humanas. ¿Fue filósofo, historiador o sociólogo? Foucault confesó que si hubiera tenido que definirse a sí mismo no hubiera sabido cómo hacerlo: "À vrai dire, je ne suis pas philosophe. Je ne fais pas de la philosophie dans ce que je fais" (Calderon 2003). Foucault se movió entre disciplinas y definió su obra como una historia crítica del pensamiento. Sus historias fueron más allá del análisis puramente histórico. Foucault no habla de hechos, sino de interpretaciones. Este rechazo por toda verdad general le acerca inevitablemente al escepticismo.

Savater define a Foucault como "un gran escritor, un estilista singular, un finísimo y minucioso analista de ideas y detalles profundos" (2009, p. 293) y G. Deleuze define a Foucault como archivista y cartógrafo. ${ }^{30} \mathrm{M}$. Belhasseb asevera que Foucault fue un escéptico nietzcheano situado en las antípodas de Heidegger..$^{31}$ A pesar de que la obra de Foucault sea inclasificable, podemos encontrar tres grandes ejes en su obra: la filosofia, la historia y las ciencias humanas (Gros 2012). A. Veinstein (2008) sostiene que Foucault no fue un pensador nihilista ni subjetivista ni relativista,

\section{Things, 1970}

27 "Leur incompatibilité a été un des traits fondamentaux de notre pensée" (Foucault 2013, p. 350).

28 "A partir du XIXe siècle, la littérature remet au jour le langage en son être" (Foucault 2013, p. 59).

29 "Être esclave de soi-même ... crest la plus grave, la plus lourde ... de toutes les servitudes" (Calderon 2003).

30 "Foucault n'a jamais pris l'écriture comme un but, comme une fin. C'est même cela qui en fait un gran écrivain" (Deleuze 2004, p. 3I).

3I “Foucault n'était pas un penseur structuraliste, comme on a coutume de le croire, mais un Sceptique. Non un nihiliste, mais un nietzschéen plus de gauche que de droite. Le sceptique Foucault, comme Montaigne, et aux antipodes de Heidegger, pensait que 'nous n'avons aucune communication à l'Être"' (Belhasseb 20I7). 
sino un pensador escéptico. Veyne asegura que Foucault fue un escéptico, pero un escéptico muy original.

Para Foucault, la relación entre subjetividad y verdad fue siempre un problema: "J'ai cherché à savoir comment le sujet humain entrait dans des jeux de vérité" (Zarka 2002, p. 255). Estos juegos de verdad pueden tomar la forma de modelos científicos o pueden encontrarse en las instituciones y prácticas de control de las que habla Foucault en Les mots et les choses. Foucault declaró que su proyecto consistía solamente en definir las versiones múltiples de la relación entre el sujeto humano y los "jeux de vérité" (Zarka 2002, p. 255). Nietzsche afirmó en Menschliches, Allzumenschliches que no hay hechos eternos ni verdades absolutas, y que la falta de sentido histórico es el pecado original de todos los filósofos. ${ }^{32}$ Quiniou (2014) asevera que el escepticismo de Foucault va más allá de su teoría del conocimiento (puesto que Foucault también negó una moral universal). Es imposible escapar de las verdades temporales: estamos hundidos en la historia.

La obra de Foucault no cesa de desbordar la filosofía (Halpern 2013). Foucault fue un pensador que se interesó por la historia como problema filosófico. ${ }^{33}$ A Foucault no solamente le atrajo la historia del pasado sino también la del futuro. Foucault no se definió ni como historiador ni como filósofo, sino como un pensador escéptico. Su escepticismo es una crítica del conocimiento. Esta crítica histórica es a veces también una crítica política. Foucault dudó de las verdades generales, de las verdades de cada época y de las verdades intemporales (Veyne 20I0).

En una entrevista a Foucault (Veyne 20I0), veinticinco días antes de su muerte, le preguntaron si él, siendo un pensador que no afirmaba ninguna verdad universal, se consideraba un escéptico; Foucault respondió: "Absolument".

32 "es giebt keine ewigen Thatsachen: sowie es keine absoluten Wahrheiten giebt. Demnach ist das historische Philosophiren von jetzt ab nöthig und mit ihm die Tugend der Bescheidung" (Nietzsche, KSA 2: I, $\$ 2$, p. 25).

33 "Derrière les mots, $M$. Foucault montre comment pratiques et discours produisent une autre réalité. Ainsi, pour M. Foucault lui même cette question sur deux disciplines se trouvent interrogées et mélées dans chacun de ces ouvrages" (Farge 2013, p. 90). 


\section{Referencias bibliográficas}

Adler, L., Boucheron, P., Gros F. y Defert D. (20I6, 25 de marzo). La trajectoire philosophique et politique de Michel Foucault. France Culture. https://www.franceculture.fr/emissions/hors-champs/michel-foucault-55-la-trajectoire-philosophique-et-politique-de-michel

Belhasseb, M. (20I7, 2 de julio). Le Samouraï de la Pensée. L'Est Républicain Algère. http://www.lestrepublicain.com/index.php/societe/ item/90oI755-le-samourai-de-la-pensee

Calderon, P. (Director). (2003). Michel Foucault par lui-même [Documental]. Francia: ARTE France.

Deleuze, G. (2004). Foucault. Francia: Les Éditions de Minuit. 1986.

Dumayet, P. (1966, I5 de junio). Michel Foucault à propos du livre "Les mots et les choses". Ina Fr. http://www.ina.fr/video/Io5059752

Eribon, D. (20II). Michel Foucault. Paris: Éditions Flammarion.

Farge, A. (20I3). Foucault et l'histoire. Entretien avec Arlette Farge. En C. Halpern (Ed.), Pensées rebelles. Foucault, Derrida, Deleuze (pp. 90-92). Paris: Éditions Sciences Humaines.

Foucault, M. (2013). Les mots et les choses. Une archéologie des sciences humaines. France: Gallimard. 1966.

(2012a). Histoire de la sexualité, vol. I. La volonté de savoir. France: Gallimard. 1976.

(2012b). L’archéologie du savoir. France: Gallimard. 1969.

Freud, S. (2013). Der Mann Moses und die monotheistische Religion. Schriften über Religion. Frankfurt am Main: Fisher Taschenbuch Verlag GmbH. 1975.

(20I2). Totem und Tabu. Frankfurt am Main: Fischer. 199I.

(20I0). Das Unbehagen in der Kultur. Stuttgart: Reclam.

(2007). Massenpsychologie und Ich-Analyse / Die Zukunft einer Il-

lusion. Frankfurt am Main: Fisher Taschenbuch Verlag GmbH. 1993.

Gros, F. (20I2). Michel Foucault. Paris: PUF. 1996.

Gutting, G. (2005a). Foucault. A Very Short Introduction. New York: Oxford University Press Inc.

- (2005b). Foucault and the History of Madness. En G. Gutting (Ed.), The Cambridge Companion to Foucault (2nd Edition) (pp. 49-73). UK: Cambridge University Press.

Halpern, C. (2013). Michel Foucault. L'insoumis. En C. Halpern (Ed.), Pensées rebelles. Foucault, Derrida, Deleuze (pp. 29-32). Paris: Éditions Sciences Humaines. 
Lahire, B. (2013). Quel apport pour la sociologie? En C. Halpern (Ed.), Pensées rebelles. Foucault, Derrida, Deleuze (pp. 8I-89). Paris: Éditions Sciences Humaines.

Lallement, M. (2013). La quête inachevée de Michel Foucault. En C. Halpern (Ed.), Pensées rebelles. Foucault, Derrida, Deleuze (pp. 33-40). Paris: Éditions Sciences Humaines.

Marx, K. (2016). Zur Kritik der Hegel'schen Rechtsphilosophie. En Karl Marx. Friedrich Engels. Gesammelte Werke. Ed. Kurt Lhotzky, Köln, Anaconda Verlag, pp. 12-28.

Nietzsche, F. (20II). Die fröhliche Wissenschaft, KSA 3. Berlín: Deutscher Taschenbuch Verlag. 1967-1977, 1988. (1988). Also sprach Zarathustra I-IV, KSA 4. Berlin: Deutscher Taschenbuch Verlag. 1967-1977. (1988). Der Fall Wagner. Götzen-Dämmerung. Der Antichrist. Ecce homo. Dionysos-Dthyramben. Nietzsche contra Wagner, KSA 6. Berlin: Deutscher Taschenbuch Verlag. 1967-1977.

_ (1967-1977). Menschliches, Allzumenschliches. Ein Buch für freie Geister, I - II, KSA 2. Berlin, Deutscher Taschenbuch Verlag.

Oñate, T. (2010). El retorno teológico-político de la inocencia. Los hijos de Nietzsche en la postmodernidad II. Madrid: Dykinson.

Ortega y Gasset, J. (2004-20IO). Obras Completas, Io volúmenes. Madrid: Taurus y Fundación Ortega y Gasset.

Piaget, J. (1974, 1968). Le structuralisme, Paris: PUF.

Pol-Droit, R. (2008, 20 de marzo). Paul Veyne: portrait de l'archéologue en sceptique. Le Monde. https://www.lemonde.fr/livres/article/2008/03/20/paul-veyne-portrait-de-l-archeologue-en-sceptique_IO2533I_326o.html

Quiniou, Y. (2009). Foucault, penseur sceptique. La pensée (359). Paris: Fondation Gabriel Péri. http://lafauteadiderot.net/Foucault-penseur-sceptique

- (2014, octubre). Démythifier Foucault. La Revue du projet (40). http://projet.pcf.fr/61207

Rambeau, F. (2006). Dossier. Le texte en perspective. En Foucault, M., La volonté de savoir. Droit de mort et pouvoir sur la vie (pp. 52-I24). France: Folioplus Philosophie.

Revel, J. (2009). Le vocabulaire de Foucault. Paris: Ellipses.

Savater, F. (2009). La aventura de pensar. Barcelona: Debolsillo. 2008.

Sabot, P. (2006). Lire Les mots et les choses de Michel Foucault. Paris: PUF. 
Veinstein A. y Veyne P. (2008, I3 de junio). Du jour au lendemain, Paul Veyne / Foucault (France Culture). http://www.fabriquedesens.net/ Paul-Veyne-Foucault-dans-Du-jour

Veyne, O. (20I0). Foucault. Sa pensée, sa personne. Paris: Éditions Grasset \& Fasquelle.

Zarka, Y. (2002). Foucault et l'idée d'une histoire de la subjectivité: le moment moderne. Archives de Philosophie, 2(2), 255-267. https://doi. org/I0.3917/aphi.652.0255 